Thomas Fraunholz, Ronald H. W. Hoppe*, and Malte Peter

\title{
Convergence analysis of an adaptive interior penalty discontinuous Galerkin method for the biharmonic problem
}

\begin{abstract}
For the biharmonic problem, we study the convergence of adaptive $C^{0}$-Interior Penalty Discontinuous Galerkin ( $C^{0}$-IPDG) methods of any polynomial order. We note that $C^{0}$-IPDG methods for fourth order elliptic boundary value problems have been suggested in $[9,17]$, whereas residual-type a posteriori error estimators for $C^{0}$-IPDG methods applied to the biharmonic equation have been developed and analyzed in $[8,18]$. Following the convergence analysis of adaptive IPDG methods for second order elliptic problems [6], we prove a contraction property for a weighted sum of the $C^{0}$-IPDG energy norm of the global discretization error and the estimator. The proof of the contraction property is based on the reliability of the estimator, a quasi-orthogonality result, and an estimator reduction property. Numerical results are given that illustrate the performance of the adaptive $C^{0}$-IPDG approach.
\end{abstract}

Keywords: $C^{0}$-interior penalty discontinuous Galerkin method, biharmonic equation, residual type a posteriori error estimator, quasioptimal convergence.

MSC 2010: 65N30, 65N50, 35J35

DOI: $10.1515 /$ jnma-2015-0021

Received October 12, 2013; accepted June 15, 2014

For second order elliptic boundary value problems, adaptive finite element methods (AFEM) are well established numerical tools that have been intensively studied in the literature (cf., e.g., $[1,3,4,16,25,28]$ and the references therein). The convergence analysis of AFEM for conforming discretizations has been initiated in [14] (cf. also [24]) with the most far reaching result so far given in [13]. Nonconforming discretizations based on the lowest order Crouzeix-Raviart elements have been addressed in [11], whereas for Interior Penalty Discontinuous Galerkin (IPDG) methods we refer to [6]. However, considerably less work has been devoted to AFEM for nonconforming discretizations of fourth order elliptic boundary value problems. As far as IPDG approaches are concerned, $C^{0}$-IPDG methods have been suggested in [15] (cf. also [30]) and subsequently analyzed in [9] focusing on an a priori error analysis. An a posteriori error analysis of quadratic $C^{0}$-IPDG methods based on residual-type a posteriori error estimators has been performed in [8], however, without addressing the issue of convergence.

The purpose of this contribution is to provide a convergence analysis of $C^{0}$-IPDG methods of any polynomial order for the biharmonic problem. The residual a posteriori error estimator consists of element and edge residuals and is a generalization to arbitrary polynomial degree $k \geqslant 2$ of the one considered in [8] for the case $k=2$. The reliability of the estimator can be shown by similar techniques as in [8]. Together with the standard estimator reduction for Dörfler marking (Lemma 4.1) and a quasi-orthogonality result (Theorem 5.3) this results in a contraction property for a weighted sum of the $C^{0}$-IPDG energy norm of the global discretization error and the estimator (Theorem 6.1). We note that in case of IPDG approximations of second order elliptic boundary value problems a contraction property for the IPDG energy norm of the error has been established in $[19,22]$ based on the reliability of the estimator, its local efficiency up to data oscillations, as well as a quasi-orthogonality property. The proof of the local efficiency relies on the interior node property. In contrast to these results, the contraction property which will be established here does neither require the

Thomas Fraunholz: Inst. of Math., University of Augsburg, D-86159 Augsburg, Germany

*Corresponding Author: Ronald H. W. Hoppe: Dept. of Math., University of Houston, Houston TX 77204-3008, U.S.A. Inst. of Math., University of Augsburg, D-86159 Augsburg, Germany. E-mail: rohop@math.uh.edu

Malte Peter: Inst. of Math. and Augsburg Centre for Innovative Technologies, Univ. of Augsburg, D-86159 Augsburg, Germany 
interior node property nor does it involve marking by data oscillations. Although the basic ingredients for the proof (reliability, estimator reduction, and quasi-orthogonality) are the same as in [6], their realizations are not straightforward and require to take into account the particular structure of the estimator. To our best knowledge this is the first contribution containing numerical results for high order IPDG approximations of the biharmonic problem that confirm the theoretically achievable quasi-optimal convergence rates.

\section{$1 C^{0}$-interior penalty Discontinuous Galerkin method}

Let $\Omega \subset \mathbb{R}^{2}$ be a bounded polygonal domain with boundary $\Gamma=\partial \Omega$. For a given function $f \in L^{2}(\Omega)$ we consider the biharmonic problem

$$
\begin{array}{cc}
\Delta^{2} u=f & \text { in } \Omega \\
u=\frac{\partial u}{\partial n}=0 & \text { on } \Gamma .
\end{array}
$$

We use standard notation from Lebesgue and Sobolev space theory [27]. In particular, $(\cdot, \cdot)_{0, \Omega}$ and $\|\cdot\|_{0, \Omega}$ stand for the inner product on $L^{2}(\Omega)$ and the associated norm. Moreover, $H^{k}(\Omega), k \in \mathbb{N}$, refers to the Sobolev space with norm $\|\cdot\|_{k, \Omega}$ and seminorm $|\cdot|_{k, \Omega}$, whereas $H_{0}^{k}(\Omega)$ denotes the closure of $C_{0}^{\infty}(\Omega)$ with respect to the topology induced by $\|\cdot\|_{k, \Omega}$. The Sobolev spaces with broken index $s \in \mathbb{R}_{+}$can be defined by interpolation and are referred to as $H^{s}(\Omega)$.

A weak formulation of (1.1) requires the computation of $u \in V:=H_{0}^{2}(\Omega)$ such that

$$
a(u, v)=(f, v)_{0, \Omega}, \quad v \in V
$$

where the bilinear form $a(\cdot, \cdot)$ is given by

$$
a(v, w)=\left(D^{2} v, D^{2} w\right)_{0, \Omega}:=\sum_{|\beta|=2}\left(D^{\beta} v, D^{\beta} w\right)_{0, \Omega}, \quad v, w \in V .
$$

Let $\mathcal{T}_{h}(\Omega)$ be a geometrically conforming simplicial triangulation of $\Omega$. For $D \subseteq \bar{\Omega}$, we denote by $\varepsilon_{h}(D)$ the set of edges of $\mathcal{T}_{h}(\Omega)$ in $D$. For $T \in \mathcal{T}_{h}(\Omega)$ and $E \in \mathcal{E}_{h}(\bar{\Omega})$ we denote by $h_{T}$ and $h_{E}$ the diameter of $T$ and the length of $E$, and we set $h:=\max \left\{h-T \mid T \in \mathcal{T}_{h}(\Omega)\right\}$. For two quantities $A$ and $B$ we write $A \leqslant B$, if there exists a constant $C>0$ independent of $h$ such that $A \leqslant C B$.

Denoting by $P_{k}(T), k \in \mathbb{N}$, the linear space of polynomials of degree $\leqslant k$ on $T$, for $k \geqslant 2$ we refer to

$$
V_{h}:=\left\{v_{h} \in H_{0}^{1}(\Omega)\left|v_{h}\right|_{T} \in P_{k}(T), T \in \mathcal{T}_{h}(\Omega)\right\}
$$

as the finite element space of Lagrangian finite elements of type $k$ (cf., e.g., [7]). For $D \subseteq \bar{\Omega}$, we denote by $\mathcal{N}_{h}(D)$ as the set of nodal points in $D$ such that any $v_{h} \in V_{h}$ is uniquely determined by its degrees of freedom $v_{h}(a), a \in \mathcal{N}_{h}(\Omega)$.

We note that $V_{h} \not \subset V$ and hence, $V_{h}$ is a nonconforming finite element space for the approximation of the biharmonic problem (1.2). In particular, for $v_{h} \in V_{h}$ the normal derivative $\partial v_{h} / \partial n$ exhibits jumps across interior edges $E \in \mathcal{E}_{h}^{\Omega}$. After numbering of the elements $T \in \mathcal{T}_{h}(\Omega)$, for $E \in \mathcal{E}_{h}(\Omega), E=T_{i} \cap T_{j}, i>j$, we set $T_{E}^{+}:=T_{i}, T_{E}^{-}:=T_{j}$, and for $E \in \mathcal{E}_{h}^{\Gamma}, E=T_{\ell} \cap \Gamma$, we set $T_{E}:=T_{\ell}$. Then, for $1 \leqslant v \leqslant 2$ we define averages and jumps according to

$$
\begin{aligned}
& \left\{\frac{\partial^{v} v_{h}}{\partial n^{v}}\right\}_{E}:=\left\{\begin{array}{lr}
\frac{1}{2}\left(\left.\frac{\partial^{v} v_{h}}{\partial n^{v}}\right|_{E \cap T_{E}^{+}}+\left.\frac{\partial^{v} v_{h}}{\partial n^{v}}\right|_{E \cap T_{E}^{-}}\right), & E \in \mathcal{E}_{h}(\Omega) \\
\left.\frac{\partial^{v} v_{h}}{\partial n^{v}}\right|_{E \cap T_{E}}, & E \in \mathcal{E}_{h}(\Gamma)
\end{array}\right. \\
& {\left[\frac{\partial^{v} v_{h}}{\partial n^{v}}\right]_{E}:=\left\{\begin{array}{lr}
\left.\frac{\partial^{v} v_{h}}{\partial n^{v}}\right|_{E \cap T_{E}^{+}}-\left.\frac{\partial^{v} v_{h}}{\partial n^{v}}\right|_{E \cap T_{E}^{-}}, & E \in \mathcal{E}_{h}(\Omega) \\
\left.\frac{\partial^{v} v_{h}}{\partial n^{v}}\right|_{E \cap T_{E}}, & E \in \mathcal{E}_{h}(\Gamma)
\end{array}\right.}
\end{aligned}
$$


where $n$ is the unit normal vector on $E$ pointing in the direction from $T_{E}^{-}$to $T_{E}^{+}$for $E \in \mathcal{E}_{h}(\Omega)$ and the exterior normal vector for $E \in \mathcal{E}_{h}(\Gamma)$.

We further refer to $M_{h}\left(\mathcal{T}_{h}(\Omega) ; \mathbb{R}^{2 \times 2}\right)$ as the set of matrix-valued functions on $\mathcal{T}_{h}(\Omega)$ such that for $W_{h} \in$ $M_{h}\left(\mathcal{T}_{h}(\Omega) ; \mathbb{R}^{2 \times 2}\right)$ the restriction $\left.W_{h}\right|_{T}, T \in \mathcal{T}_{h}(\Omega)$, is a $2 \times 2$ matrix with entries that are polynomials of order $k$.

Given a penalty parameter $\alpha>1$, the $C^{0}$-IPDG method for the approximation of (1.2) requires the computation of $u_{h} \in V_{h}$ such that

$$
a_{h}^{I P}\left(u_{h}, v_{h}\right)=\left(f, v_{h}\right)_{0, \Omega}, \quad v_{h} \in V_{h} .
$$

Here, the mesh-dependent bilinear form $a_{h}^{I P}(\cdot, \cdot): V_{h} \times V_{h} \rightarrow \mathbb{R}$ is given according to

$$
\begin{aligned}
a_{h}^{I P}\left(v_{h}, w_{h}\right):= & \sum_{T \in \mathcal{T}_{h}(\Omega)}\left(D^{2} v_{h}, D^{2} w_{h}\right)_{0, T}+\sum_{E \in \mathcal{E}_{h}(\Omega)}\left(\left\{\frac{\partial^{2} v_{h}}{\partial n^{2}}\right\}_{E},\left[\frac{\partial w_{h}}{\partial n}\right]_{E}\right)_{0, E} \\
& +\sum_{E \in \mathcal{E}_{h}(\Omega)}\left(\left[\frac{\partial v_{h}}{\partial n}\right]_{E},\left\{\frac{\partial^{2} w_{h}}{\partial n^{2}}\right\}_{E}\right)_{0, E}+\alpha \sum_{E \in \mathcal{E}_{h}(\bar{\Omega})} h_{E}^{-1}\left(\left[\frac{\partial v_{h}}{\partial n}\right]_{E},\left[\frac{\partial w_{h}}{\partial n}\right]_{E}\right)_{0, E} .
\end{aligned}
$$

We note that $a_{h}^{I P}(\cdot, \cdot)$ is not well defined for $v, w \in V$ which can be cured in terms of a lifting operator $L$ : $L^{2}\left(\mathcal{E}_{h}(\bar{\Omega}), \mathbb{R}^{2}\right) \rightarrow M_{h}\left(\mathcal{T}_{h}(\Omega) ; \mathbb{R}^{2 \times 2}\right)$ given by

$$
\left(L(q), W_{h}\right)_{0, \Omega}:=\sum_{E \in \mathcal{E}_{h}(\bar{\Omega})}\left([n \cdot q]_{E},\left\{n \cdot W_{h} n\right\}_{E}\right)_{0, E}
$$

for $W_{h} \in M_{h}\left(\mathcal{T}_{h}(\Omega) ; \mathbb{R}^{2 \times 2}\right)$. We refer to [20, 21] for lifting operators in case of DG approximations of second order problems and to [18] for a lifting operator associated with IPDG approximations of the biharmonic problem. The bilinear form $a_{h}^{I P}(\cdot, \cdot)$ can be extended to $V+V_{h}$ by means of

$$
\begin{aligned}
a_{h}^{I P}(v, w):= & \sum_{T \in \mathcal{T}_{h}(\Omega)}\left(D^{2} v, D^{2} w\right)_{0, T}+\sum_{T \in \mathcal{T}_{h}(\Omega)}\left(L(\nabla w), D^{2} v\right)_{0, T} \\
& +\sum_{T \in \mathcal{T}_{h}(\Omega)}\left(L(\nabla v), D^{2} w\right)_{0, T}+\alpha \sum_{E \in \mathcal{E}_{h}(\bar{\Omega})} h_{E}^{-1}\left(\left[\frac{\partial v}{\partial n}\right]_{E},\left[\frac{\partial w}{\partial n}\right]_{E}\right)_{0, E}
\end{aligned}
$$

where with a slight abuse of notation we have also used $a_{h}^{I P}(\cdot, \cdot)$ for that extension.

The lifting operator satisfies the following stability estimate.

Theorem 1.1. Let $L: L^{2}\left(\mathcal{E}_{h}(\bar{\Omega}), \mathbb{R}^{2}\right) \rightarrow M_{h}\left(\mathcal{T}_{h}(\Omega) ; \mathbb{R}^{2 \times 2}\right)$ be the lifting operator as given by (1.8). Then, there exists a positive constant $C_{L}$, depending only on the local geometry of the triangulation and on the polynomial order $k$, such that there holds

$$
\|L(q)\|_{0, \Omega}^{2} \leqslant C_{L} \sum_{E \in \mathcal{E}_{h}(\bar{\Omega})} h_{E}^{-1}\left\|[n \cdot q]_{E}\right\|_{0, E}^{2}, \quad q \in L^{2}\left(\mathcal{E}_{h}(\bar{\Omega}), \mathbb{R}^{2}\right) .
$$

Proof. For $q \in L^{2}\left(\mathcal{E}_{h}(\bar{\Omega}), \mathbb{R}^{2}\right)$ and $W_{h} \in M_{h}\left(\mathcal{T}_{h}(\Omega) ; \mathbb{R}^{2 \times 2}\right)$ we have

$$
\|L(q)\|_{0, \Omega}=\sup _{\left\|W_{h}\right\|_{0, \Omega} \leqslant 1}\left|\left(L(q), W_{h}\right)_{0, \Omega}\right| .
$$

In view of (1.8) we find

$$
\left|\left(L(q), W_{h}\right)_{0, \Omega}\right| \leqslant\left(\sum_{E \in \mathcal{E}_{h}(\bar{\Omega})}\left\|[n \cdot q]_{E}\right\|_{0, E}^{2}\right)^{1 / 2}\left(\sum_{T \in \mathcal{T}_{h}(\Omega)}\left\|n_{\partial T} \cdot W_{h} n_{\partial T}\right\|_{0, \partial T}^{2}\right)^{1 / 2}
$$

where $n_{\partial T}$ is the exterior unit normal on $\partial T$. Then, the trace inequality (cf., e.g., [29]):

$$
\left\|n_{\partial T} \cdot W_{h} n_{\partial T}\right\|_{0, \partial T} \lesssim k h_{T}^{-1 / 2}\left\|W_{h}\right\|_{0, T}, \quad T \in \mathcal{T}_{h}(\Omega)
$$

gives the assertion. 
On $V+V_{h}$ we introduce the mesh-dependent $C^{0}$-IPDG norm

$$
\|v\|_{2, h, \Omega}^{2}:=\sum_{T \in \mathcal{T}_{h}(\Omega)}|v|_{2, T}^{2}+\sum_{E \in \mathcal{E}_{h}(\bar{\Omega})} \frac{\alpha}{h_{E}}\left\|\left[\frac{\partial v}{\partial n}\right]_{E}\right\|_{0, E}^{2}, \quad v \in V+V_{h}
$$

where $|\cdot|_{2, T}^{2}$ stands for

$$
|\cdot|_{2, T}^{2}:=\sum_{|\beta|=2}\left\|D^{\beta} \cdot\right\|_{0, T}^{2}, \quad T \in \mathcal{T}_{h} .
$$

It has been shown in [9] that for sufficiently large penalty parameter $\alpha$ there exists a positive constant $\gamma<1$ such that

$$
a_{h}^{I P}(v, v) \geqslant \gamma\|v\|_{2, h, \Omega}^{2}, \quad v \in V+V_{h}
$$

whereas there exists a constant $C_{1}>1$ such that for any $\alpha \geqslant 1$

$$
a_{h}^{I P}(v, w) \leqslant C_{1}\|v\|_{2, h, \Omega}\|w\|_{2, h, \Omega}, \quad v, w \in V+V_{h} .
$$

In particular, it follows from (1.13) and (1.14) that (1.6) admits a unique solution $u_{h} \in V_{h}$.

\section{Residual-type a posteriori error estimator and its reliability}

For adaptive mesh refinement we consider the residual-type a posteriori error estimator

$$
\eta_{h}^{2}:=\sum_{T \in \mathcal{T}_{h}} \eta_{T}^{2}+\sum_{E \in \mathcal{E}_{h}(\Omega)} \eta_{E}^{2}+\sum_{E \in \mathcal{E}_{h}(\bar{\Omega})} \eta_{E, c}^{2}
$$

where the element residuals $\eta_{T}, T \in \mathcal{T}_{h}(\Omega)$, and the edge residuals $\eta_{E}, E \in \mathcal{E}_{h}(\Omega)$, as well as $\eta_{E, c}, E \in \mathcal{E}_{h}(\bar{\Omega})$, are given by

$$
\begin{aligned}
\eta_{T}^{2} & :=h_{T}^{4}\left\|f-\Delta^{2} u_{h}\right\|_{0, T}^{2}, \quad T \in \mathcal{T}_{h}(\Omega) \\
\eta_{E}^{2} & :=h_{E}\left\|\left[\frac{\partial^{2} u_{h}}{\partial n^{2}}\right]_{E}\right\|_{0, E}^{2}+h_{E}^{3}\left\|\left[\frac{\partial}{\partial n} \Delta u_{h}\right]_{E}\right\|_{0, E}^{2}, \quad E \in \mathcal{E}_{h}(\Omega) \\
\eta_{E, c}^{2} & :=\alpha \hat{\eta}_{E, c}^{2}, \quad \hat{\eta}_{E, c}^{2}:=h_{E}^{-1}\left\|\left[\frac{\partial u_{h}}{\partial n}\right]_{E}\right\|_{0, E}^{2}, \quad E \in \mathcal{E}_{h}(\bar{\Omega}) .
\end{aligned}
$$

For notational convenience we set

$$
\eta_{h, c}^{2}:=\alpha \hat{\eta}_{h, c}^{2}, \quad \hat{\eta}_{h, c}^{2}:=\sum_{E \in \varepsilon_{h}(\bar{\Omega})} \eta_{E, c}^{2} .
$$

The term $\hat{\eta}_{h, c}$ represents an upper bound for the consistency error

$$
\inf _{v_{h} \in V_{h}^{c}} a_{h}^{I P}\left(u_{h}-v_{h}, u_{h}-v_{h}\right)
$$

where $V_{h}^{c} \subset H_{0}^{2}(\Omega)$ stands for the $C^{1}$ conforming finite element space generated by the Argyris elements of the so-called TUBA family [2]. We use the enrichment operator (or recovery operator) $E_{h}: V_{h} \rightarrow V_{h}^{c}$ from [9] which is defined by averaging according to

$$
N\left(E_{h} v_{h}\right)=\left|\omega_{h}^{p}\right|^{-1} \sum_{T \in \omega_{h}^{p}}\left(\left.N v_{h}\right|_{T}\right), \quad v_{h} \in V_{h}
$$


where $p$ is any nodal point for $V_{h}^{c}, N$ is any nodal variable at $p$, and $\omega_{h}^{p}:=\bigcup\left\{T \in \mathcal{T}_{h}(\Omega) \mid\{p\} \cap \mathcal{N}_{h}(T) \neq \varnothing\right\}$. It follows from the mapping properties of $E_{h}$ established in [9] that there exists a constant $C_{n c}>0$, depending only on the local geometry of $\mathcal{T}_{h}(\Omega)$, such that

$$
\inf _{v_{h} \in V_{h}^{c}} a_{h}^{I P}\left(u_{h}-v_{h}, u_{h}-v_{h}\right) \leqslant a_{h}^{I P}\left(u_{h}-E_{h}\left(u_{h}\right), u_{h}-E_{h}\left(u_{h}\right)\right) \leqslant C_{n c} \hat{\eta}_{h, c}^{2} .
$$

The following result shows that $\eta_{h}^{2}$ provides an upper bound for the IPDG energy norm of the discretization error $u-u_{h}$. It can be shown by using similar techniques as in [8].

Theorem 2.1. Let $u \in V$ and $u_{h} \in V_{h}$ be the unique solution of (1.2) and (1.6), and let $\eta_{h}$ be given by (2.1) and (2.2). Then, there exists a constant $C_{R}>0$, depending only on the local geometry of $\mathcal{T}_{h}$ and on $k$, such that

$$
a_{h}^{I P}\left(u-u_{h}, u-u_{h}\right) \leqslant C_{R} \eta_{h}^{2} .
$$

\section{Refinement strategy and estimator reduction}

As a marking strategy for adaptive refinement we use Dörfler marking [14]. To this end, we reformulate the estimator $\eta_{h}$ (cf. (2.1)) according to

$$
\begin{aligned}
\eta_{h}= & \left(\sum_{T \in \mathcal{T}_{h}(\Omega)} \hat{\eta}_{T}^{2}\right)^{1 / 2} \\
\hat{\eta}_{T}^{2}:= & h_{T}^{4}\left\|f-\Delta^{2} u_{h}\right\|_{0, T}^{2}+\frac{1}{2} \sum_{E \in \mathcal{E}_{h}(\partial T \cap \Omega)}\left(\alpha h_{E}^{-1}\left\|\left[\frac{\partial u_{h}}{\partial n}\right]_{E}\right\|_{0, E}^{2}\right. \\
& \left.+h_{E}\left\|\left[\frac{\partial^{2} u_{h}}{\partial n^{2}}\right]_{E}\right\|_{0, E}^{2}+h_{E}^{3}\left\|\left[\frac{\partial}{\partial n} \Delta u_{h}\right]_{E}\right\|_{0, E}^{2}\right)+\sum_{E \in \mathcal{E}_{h}(\partial T \cap \Gamma)} \alpha h_{E}^{-1}\left\|\left[\frac{\partial u_{h}}{\partial n}\right]_{E}\right\|_{0, E}^{2} .
\end{aligned}
$$

Then, given a constant $0<\Theta<1$, we compute a set $\mathcal{M}$ of elements $T \in \mathcal{T}_{h}(\Omega)$ such that

$$
\Theta \eta_{h}^{2} \leqslant \sum_{T \in \mathcal{M}} \hat{\eta}_{T}^{2} .
$$

After having determined the set $\mathcal{M}$, a refined triangulation is generated by a recursive application of newest vertex bisection. Assuming a conforming initial triangulation that satisfies a certain labeling condition, this leads to quasi-optimal cardinality (cf. Section 4 in [13] and Subsection 3.4 in [6]). In particular, there exist constants $0<\beta_{1}<\beta_{2}$, depending only on the initial triangulation, such that for each triangle $T$ of refinement level $\ell$ it holds $\beta_{1} 2^{-\ell / 2} \leqslant h_{T} \leqslant \beta_{2} 2^{-\ell / 2}$. Hence, if $\mathcal{T}_{h}(\Omega)$ is obtained from $\mathcal{T}_{H}(\Omega)$ by newest vertex bisection, for $T \in \mathcal{T}_{H}(\Omega)$ and $T \supset T^{\prime} \in \mathcal{T}_{h}(\Omega)$ we have

$$
\varkappa_{1} h_{T^{\prime}} \leqslant H_{T} \leqslant \varkappa_{2} h_{T^{\prime}}
$$

where $\varkappa_{1}:=2^{1 / 2} \beta_{1} / \beta_{2}$ and $\varkappa_{2}:=2^{1 / 2} \beta_{2} / \beta_{1}$.

As in [13] (cf. also [6]), we can prove the following estimator reduction property.

Lemma 3.1. Let $\mathcal{T}_{h}(\Omega)$ be a simplicial triangulation obtained by refinement from $\mathcal{T}_{H}(\Omega)$, let $u_{h} \in V_{h}, u_{H} \in V_{H}$, and $\eta_{h}, \eta_{H}$ be the associated $C^{0}$-IPDG solutions and error estimators, respectively, and let $\Theta>0$ be the universal constant from (3.1). Then, for any $\tau>0$ there exists a constant $C_{\tau}>1$, depending only on the local geometry of the triangulations and on $k$, such that for $\varkappa(\Theta):=(1+\tau)\left(1-2^{-1 / 2}\right) \Theta$ there holds

$$
\eta_{h}^{2} \leqslant \varkappa(\Theta) \eta_{H}^{2}+C_{\tau}\left\|u_{h}-u_{H}\right\|_{2, h, \Omega}^{2} .
$$


Proof. By definition of $\eta_{h}$ and taking into account the inverse estimates

$$
\begin{aligned}
& \left\|\Delta^{2}\left(u_{h}-u_{H}\right)\right\|_{0, T} \leqslant C_{\text {inv }}^{(1)} k^{4} h_{T}^{-2}\left\|D^{2}\left(u_{h}-u_{H}\right)\right\|_{0, T}, \quad T \in \mathcal{T}_{h}(\Omega) \\
& \left\|\frac{\partial^{2}\left(u_{h}-u_{H}\right)}{\partial n_{E \cap \partial T}^{2}}\right\|_{0, E} \leqslant C_{\text {inv }}^{(2)} k^{2} h_{E}^{-1}\left\|\frac{\partial\left(u_{h}-u_{H}\right)}{\partial n_{E \cap \partial T}}\right\|_{0, E}, \quad E \in \mathcal{E}_{h}(T) \\
& \left\|\frac{\partial}{\partial n_{E \cap \partial T}} \Delta\left(u_{h}-u_{H}\right)\right\|_{0, E} \leqslant C_{\text {inv }}^{(3)} k^{4} h_{E}^{-2}\left\|\frac{\partial\left(u_{h}-u_{H}\right)}{\partial n_{E \cap \partial T}}\right\|_{0, E}, \quad E \in \mathcal{E}_{h}(T)
\end{aligned}
$$

where $C_{\text {inv }}^{(i)}, i=1,2,3$, are positive constants, depending only on the local geometry of the triangulations, we have

$$
\begin{aligned}
& h_{T}^{2}\left\|f-\Delta^{2} u_{h}\right\|_{0, T} \leqslant h_{T}^{2}\left(\left\|f-\Delta^{2} u_{H}\right\|_{0, T}+C_{\text {inv }}^{(1)} k^{4} h_{T}^{-2}\left\|D^{2}\left(u_{h}-u_{H}\right)\right\|_{0, T}\right) \\
& h_{E}^{1 / 2}\left\|\frac{\partial^{2} u_{h}}{\partial n_{E \cap \partial T}^{2}}\right\|_{0, E} \leqslant h_{E}^{1 / 2}\left\|\frac{\partial^{2} u_{H}}{\partial n_{E \cap \partial T}^{2}}\right\|_{0, E}+C_{\text {inv }}^{(2)} k^{2} h_{E}^{-1 / 2} \| \frac{\partial\left(u_{h}-u_{H}\right)}{\partial n_{E \cap \partial T} \|_{0, E}} \\
& h_{E}^{3 / 2}\left\|\frac{\partial}{\partial n_{E \cap \partial T}} \Delta u_{h}\right\|_{0, E} \leqslant h_{E}^{3 / 2}\left\|\frac{\partial}{\partial n_{E \cap \partial T}} \Delta u_{H}\right\|_{0, E}+C_{\text {inv }}^{(3)} k^{4} h_{E}^{-1 / 2}\left\|\frac{\partial\left(u_{h}-u_{H}\right)}{\partial n_{E \cap \partial T}}\right\|_{0, E} .
\end{aligned}
$$

By an application of Young's inequality, in view of (1.11), (3.2) and observing $\alpha \geqslant 1$, from (3.4) and the marking and refinement strategy we deduce the existence of $C_{\mathrm{ER}}>1$, depending only on the local geometry of the triangulations and on $k$, such that for $\tau>0$ there holds

$$
\begin{aligned}
\eta_{h}^{2} \leqslant & (1+\tau)\left(1-2^{-1 / 2}\right) \Theta\left(\sum_{T \in \mathcal{T}_{H}(\Omega)} H_{T}^{4}\left\|f-\Delta^{2} u_{H}\right\|_{0, T}^{2}+\sum_{E \in \mathcal{E}_{H}(\Omega)}\left(H_{E}\left\|\left[\frac{\partial^{2} u_{H}}{\partial n}\right]_{E}\right\|_{0, E}^{2}+H_{E}^{3}\left\|\left[\frac{\partial}{\partial n_{E}} \Delta u_{H}\right]_{E}\right\|_{0, E}^{2}\right)\right) \\
& +\sum_{E \in \mathcal{E}_{H}(\bar{\Omega})} \alpha H_{E}^{-1}\left\|\left[\frac{\partial u_{H}}{\partial n}\right]_{E}\right\|_{0, E}^{2}+\left(1+\tau^{-1}\right) C_{\mathrm{ER}}\left\|u_{h}-u_{H}\right\|_{2, h, \Omega}^{2}
\end{aligned}
$$

which gives the assertion with $C_{\tau}:=\left(1+\tau^{-1}\right) C_{\mathrm{ER}}$.

Remark 3.1. If we choose $\tau=2^{-1 / 2}$ and observe $0<\Theta \leqslant 1$, we have

$$
\varkappa(\Theta)=\frac{1}{2} \Theta \leqslant \frac{1}{2} \text {. }
$$

\section{Quasi-orthogonality}

As a further significant ingredient of the convergence analysis, in this section we prove quasi-orthogonality of the $C^{0}$-IPDG approach. We first provide a mesh perturbation result in Subsection 4.1 and then establish quasi-orthogonality in Subsection 4.2.

\subsection{Mesh perturbation result}

In the convergence analysis of IPDG methods for second order elliptic boundary value problems, mesh perturbation results estimating the coarse mesh error in the fine mesh energy norm from above by its coarse mesh energy norm have played a central role in the convergence analysis as a prerequisite for establishing a quasi-orthogonality result (cf., e.g., [6, 19, 22]). Here, we provide the following mesh perturbation result.

Lemma 4.1. Let $\mathcal{T}_{h}(\Omega)$ be a simplicial triangulation obtained by refinement from $\mathcal{T}_{H}$. Then, there exists a constant $C_{P}>0$, depending only on the local geometry of the triangulations and on $k$, such that for any $\varepsilon>0$ and $v \in V+V_{H}$ there holds

$$
a_{h}^{I P}(v, v) \leqslant(1+\varepsilon) a_{H}^{I P}(v, v)+\frac{C_{P}}{\varepsilon}\left(\sum_{E \in \mathcal{E}_{h}(\bar{\Omega})} h_{E}^{-1}\left\|\left[\frac{\partial v}{\partial n}\right]_{E}\right\|_{0, E}^{2}+\sum_{E \in \mathcal{E}_{H}(\bar{\Omega})} H_{E}^{-1}\left\|\left[\frac{\partial v}{\partial n}\right]_{E}\right\|_{0, E}^{2}\right) .
$$


Proof. For $v \in V+V_{H}$ we have

$$
a_{h}^{I P}(v, v)=\sum_{T \in \mathcal{T}_{h}(\Omega)}\left\|D^{2} v\right\|_{0, T}^{2}+\sum_{E \in \mathcal{E}_{h}(\bar{\Omega})} \frac{\alpha}{h_{E}}\left\|\left[\frac{\partial v}{\partial n}\right]_{E}\right\|_{0, E}^{2}+2 \sum_{T \in \mathcal{T}_{h}(\Omega)}\left(L(\nabla v), D^{2} v\right)_{0, T} .
$$

Obviously, there holds

$$
\sum_{T \in \mathcal{T}_{h}(\Omega)}\left\|D^{2} v\right\|_{0, T}^{2}=\sum_{T \in \mathcal{T}_{H}(\Omega)}\left\|D^{2} v\right\|_{0, T}^{2} .
$$

Moreover, in view of (3.2) we have

$$
\sum_{E \in \mathcal{E}_{h}(\bar{\Omega})} \frac{1}{h_{E}}\left\|\left[\frac{\partial v}{\partial n}\right]_{E}\right\|_{0, E, h}^{2} \leqslant \varkappa_{2}^{-1} \sum_{E \in \mathcal{E}_{H}(\bar{\Omega})} \frac{1}{H_{E}}\left\|\left[\frac{\partial v}{\partial n}\right]_{E}\right\|_{0, E, H}^{2} .
$$

Using (4.3) and (4.4) in (4.2) and observing $\varkappa_{2}^{-1}<1$, we find

$$
\begin{aligned}
a_{h}^{I P}(v, v) & \leqslant a_{H}^{I P}(v, v)+\left(\varkappa_{2}^{-1}-1\right) \sum_{E \in \mathcal{E}_{H}(\bar{\Omega})} \frac{\alpha}{H_{E}}\left\|\left[\frac{\partial v}{\partial n}\right]_{E}\right\|_{0, E}^{2}+2 \sum_{T \in \mathcal{T}_{h}(\Omega)}\left(L(\nabla v), D^{2} v\right)_{0, T}-2 \sum_{T \in \mathcal{T}_{H}(\Omega)}\left(L(\nabla v), D^{2} v\right)_{0, T} \\
& \leqslant a_{H}^{I P}(v, v)+2 \sum_{T \in \mathcal{T}_{h}(\Omega)}\left(L(\nabla v), D^{2} v\right)_{0, T}-2 \sum_{T \in \mathcal{T}_{H}(\Omega)}\left(L(\nabla v), D^{2} v\right)_{0, T} .
\end{aligned}
$$

Using Young's inequality, (1.10), and (1.13), we find

$$
\begin{aligned}
2\left|\sum_{T \in \mathcal{T}_{h}(\Omega)}\left(L(\nabla v), D^{2} v\right)_{0, T}\right| & \leqslant 2 \sum_{T \in \mathcal{T}_{h}(\Omega)}\|L(\nabla v)\|_{0, T}\left\|D^{2} v\right\|_{0, T} \leqslant \frac{\gamma}{\varepsilon}\|L(\nabla v)\|_{0, \Omega}^{2}+\frac{\varepsilon}{2 \gamma} \sum_{T \in \mathcal{T}_{h}(\Omega)}\left\|D^{2} v\right\|_{0, T}^{2} \\
& \leqslant 2 \frac{C_{L} \gamma}{\varepsilon} \sum_{E \in \mathcal{E}_{h}(\bar{\Omega})} \frac{1}{h_{E}}\left\|\left[\frac{\partial v}{\partial n}\right]_{E}\right\|_{0, E, h}^{2}+\frac{\varepsilon}{2} a_{h}^{I P}(v, v) .
\end{aligned}
$$

Further, taking Young's inequality and (1.10) as well as (4.3) into account, it follows that

$$
2\left|\sum_{T \in \mathcal{T}_{H}(\Omega)}\left(L(\nabla v), D^{2} v\right)_{0, T}\right| \leqslant 2 \frac{C_{L} \gamma}{\varepsilon} \sum_{E \in \mathcal{E}_{H}(\bar{\Omega})} \frac{1}{H_{E}}\left\|\left[\frac{\partial v}{\partial n}\right]_{E}\right\|_{0, E, h}^{2}+\frac{\varepsilon}{2} a_{h}^{I P}(v, v) .
$$

Finally, using (4.6) and (4.7) in (4.5), we deduce (4.1) with $C_{P}:=2 C_{L} \gamma$.

\subsection{Quasi-orthogonality}

The quasi-orthogonality result can be derived using the conforming approximations $u_{H}^{c} \in V_{H}^{c}, u_{h}^{c} \in V_{h}^{c}$ of (1.2) which are given as the unique solutions of

$$
\begin{aligned}
& a\left(u_{H}^{c}, v_{H}^{c}\right)=\left(f, v_{H}^{c}\right), \quad v_{H}^{c} \in V_{H}^{c} \\
& a\left(u_{h}^{c}, v_{h}^{c}\right)=\left(f, v_{h}^{c}\right), \quad v_{h}^{c} \in V_{h}^{c} .
\end{aligned}
$$

In particular, we assume that the data of the problem, i.e., the domain $\Omega$ and the right-hand $\operatorname{side} f$, are such that the solution $u$ of (1.2) satisfies $u \in H^{2+\varkappa}(\Omega) \cap V$ for some $\varkappa>1 / 2$. Then there exists a constant $C_{a p}>0$, independent of $H$ and $h$, such that the following a priori error estimates hold true

$$
\left\|u-u_{H}^{c}\right\|_{2, \Omega} \leqslant C_{a p} H^{\varkappa}\|u\|_{2+\varkappa, \Omega}, \quad\left\|u-u_{h}^{c}\right\|_{2, \Omega} \leqslant C_{a p} h^{\varkappa}\|u\|_{2+\varkappa, \Omega} .
$$

Lemma 4.2. Let $\mathcal{T}_{h}$ be a simplicial triangulation obtained by refinement from $\mathcal{T}_{H}$, and let $u_{h} \in V_{h}, u_{H} \in V_{H}$ and $\eta_{h}, \eta_{H}$ be the $C^{0}$-IPDG solutions of (1.6) and error estimators, respectively. Moreover, let $u_{h}^{c} \in V_{h}^{c}$ and $u_{H}^{c} \in V_{H}^{c}$ be the conforming approximations of (1.2) according to (4.8). Then, for $u_{h}^{n c}:=u_{h}-u_{h}^{c}$ and $u_{H}^{n c}:=u_{H}-u_{H}^{c}$ it holds

$$
\left\|u_{h}^{n c}-u_{H}^{n c}\right\|_{2, h, \Omega}^{2} \leqslant \frac{2 C_{n c}}{\gamma \alpha}\left(\eta_{h}^{2}+\eta_{H}^{2}\right)
$$

where $C_{n c}$ is the constant from (2.4). 
Proof. Due to (1.13) we have

$$
\left\|u_{h}^{n c}-u_{H}^{n c}\right\|_{2, h, \Omega}^{2} \leqslant 2\left(\left\|u_{h}^{n c}\right\|_{2, h, \Omega}^{2}+\left\|u_{H}^{n c}\right\|_{2, h, \Omega}^{2}\right) \leqslant \frac{2}{\gamma}\left(a_{h}^{I P}\left(u_{h}^{n c}, u_{h}^{n c}\right)+a_{h}^{I P}\left(u_{H}^{n c}, u_{H}^{n c}\right)\right) .
$$

On the other hand, in view of (2.4) it holds

$$
\frac{2}{\gamma} a_{h}^{I P}\left(u_{h}^{n c}, u_{h}^{n c}\right) \leqslant \frac{2 C_{n c}}{\gamma} \hat{\eta}_{h, c}^{2} \leqslant \frac{2 C_{n c}}{\gamma \alpha} \eta_{h, c}^{2} \leqslant \frac{2 C_{n c}}{\gamma \alpha} \eta_{h}^{2} .
$$

Likewise, taking

$$
\sum_{E \in \mathcal{E}_{h}(\bar{\Omega}} \frac{1}{h_{E}}\left\|\left[\frac{\partial u_{H}^{n c}}{\partial n}\right]_{E}\right\|_{0, E}^{2} \leqslant \mathcal{\varkappa}_{2}^{-1} \sum_{E \in \mathcal{E}_{H}(\bar{\Omega})} \frac{1}{H_{E}}\left\|\left[\frac{\partial u_{H}^{n c}}{\partial n}\right]_{E}\right\|_{0, E}^{2}
$$

into account, we find

$$
\frac{2}{\gamma} a_{h}^{I P}\left(u_{H}^{n c}, u_{H}^{n c}\right) \leqslant \frac{2 \varkappa_{2}^{-1} C_{n c}}{\gamma \alpha} \eta_{H}^{2} .
$$

Noting that $\varkappa_{2}^{-1}<1$, we conclude by using (4.12) and (4.13) in (4.11).

The quasi-orthogonality result reads as follows.

Theorem 4.1. Let $\mathcal{T}_{h}$ be a simplicial triangulation obtained by refinement from $\mathcal{T}_{H}$, and let $u_{h} \in V_{h}, u_{H} \in V_{H}$ and $\eta_{h}, \eta_{H}$ be the associated $C^{0}$-IPDG solutions of (1.6) and error estimators, respectively, and let $e_{h}:=u-u_{h}$ and $e_{H}:=u-u_{H}$ be the fine and coarse mesh errors. Further, assume that (4.9) holds true. Then, for any $0<\varepsilon<1$ and sufficiently small mesh width $H$ there exists a constant $C_{Q}>0$, independent of $H$ and $h$, such that it holds

$$
a_{h}^{I P}\left(e_{h}, e_{h}\right) \leqslant(1+\varepsilon) a_{H}^{I P}\left(e_{H}, e_{H}\right)-\frac{\gamma}{4}\left\|u_{h}-u_{H}\right\|_{2, h, \Omega}^{2}+\frac{C_{Q}}{\alpha \varepsilon}\left(\eta_{h, c}^{2}+\eta_{H, c}^{2}\right) .
$$

The proof of Theorem 4.1 will be provided by a series of lemmas.

Lemma 4.3. Under the assumptions of Theorem 4.1, for any $0<\varepsilon<1$ it holds

$$
a_{h}^{I P}\left(e_{h}+u_{h}^{c}-u_{H}^{c}, e_{h}+u_{h}^{c}-u_{H}^{c}\right) \leqslant(1+\varepsilon) a_{H}^{I P}\left(e_{H}, e_{H}\right)+\left(1+\frac{4}{\varepsilon}\right) \frac{C_{P}+2 C_{1} C_{n c}}{\alpha \gamma}\left(\eta_{h, c}^{2}+\eta_{H, c}^{2}\right) .
$$

Proof. Using $u_{h}+u_{h}^{c}-u_{H}^{c}=u_{H}-u_{H}^{n c}+u_{h}^{n c}$, (1.14), and Young's inequality twice, it follows that for any $0<\varepsilon_{2}<1$ it holds

$$
\begin{aligned}
& a_{h}^{I P}\left(e_{h}+u_{h}^{c}-u_{H}^{c}, e_{h}+u_{h}^{c}-u_{H}^{c}\right)=a_{h}^{I P}\left(e_{H}-\left(u_{h}^{n c}-u_{H}^{n c}\right), e_{H}-\left(u_{h}^{n c}-u_{H}^{n c}\right)\right) \\
& \quad=a_{h}^{I P}\left(e_{H}, e_{H}\right)-2 a_{h}^{I P}\left(e_{H}, u_{h}^{n c}-u_{H}^{n c}\right)+a_{h}^{I P}\left(u_{h}^{n c}-u_{H}^{n c}, u_{h}^{n c}-u_{H}^{n c}\right) \\
& \quad \leqslant a_{h}^{I P}\left(e_{H}, e_{H}\right)+2 a_{h}^{I P}\left(e_{H}, e_{h}\right)^{1 / 2} a_{h}^{I P}\left(u_{h}^{n c}-u_{H}^{n c}, u_{h}^{n c}-u_{H}^{n c}\right)^{1 / 2}+a_{h}^{I P}\left(u_{h}^{n c}-u_{H}^{n c}, u_{h}^{n c}-u_{H}^{n c}\right) \\
& \left.\left.\quad \leqslant a_{h}^{I P}\left(e_{H}, e_{H}\right)+2 C_{1}^{1 / 2} a_{h}^{I P}\left(e_{H}, e_{H}\right)^{1 / 2} \| u_{h}^{n c}-u_{H}^{n c}\right)\left\|_{2, h, \Omega}+C_{1}\right\| u_{h}^{n c}-u_{H}^{n c}\right) \|_{2, h, \Omega}^{2} \\
& \left.\quad \leqslant\left(1+\varepsilon_{2}\right) a_{h}^{I P}\left(e_{H}, e_{H}\right)+C_{1}\left(1+\frac{1}{\varepsilon_{2}}\right) \| u_{h}^{n c}-u_{H}^{n c}\right) \|_{2, h, \Omega}^{2} .
\end{aligned}
$$

An application of Lemma 4.1 (with $0<\varepsilon_{1}<1$ ) and of Lemma 4.2 to the right-hand side of 4.16 yields

$$
\begin{aligned}
a_{h}^{I P}\left(e_{h}+u_{h}^{c}-u_{H}^{c}, e_{h}+u_{h}^{c}-u_{H}^{c}\right) \leqslant & \left(1+\varepsilon_{1}\right)\left(1+\varepsilon_{2}\right) a_{H}^{I P}\left(e_{H}, e_{H}\right) \\
& +\frac{1}{\alpha \gamma}\left(\frac{1+\varepsilon_{2}}{\varepsilon_{1}} C_{P}+2 C_{1} C_{n c}\left(1+\frac{1}{\varepsilon_{2}}\right)\right)\left(\eta_{h, c}^{2}+\eta_{H, c}^{2}\right) .
\end{aligned}
$$

Finally, choosing $\varepsilon_{1}=\varepsilon_{2}=\varepsilon / 4,0<\varepsilon<1$, in (4.17) gives the assertion.

Lemma 4.4. Under the assumptions of Theorem 4.1 it holds

$$
a_{h}^{I P}\left(e_{h}, u_{h}^{c}-u_{H}^{c}\right) \leqslant C_{a p} H^{1 / 2+\varkappa}\left(C_{1}\left\|u_{h}-u_{H}\right\|_{2, h, \Omega}^{2}+\left(2+C_{1}\right)\|u\|_{5 / 2+\varkappa, \Omega}^{2}+\|f\|_{0, \Omega}^{2}\right) .
$$


Proof. We have

$$
a_{h}^{I P}\left(e_{h}, u_{h}^{c}-u_{H}^{c}\right)=a_{h}^{I P}\left(u-E_{H}\left(u_{H}\right), u_{h}^{c}-u_{H}^{c}\right)-a_{h}^{I P}\left(u_{H}-E_{H}\left(u_{H}\right), u_{h}^{c}-u_{H}^{c}\right)+a_{h}^{I P}\left(u_{H}-u_{h}, u_{h}^{c}-u_{H}^{c}\right) .
$$

Since $E_{H}\left(u_{H}\right) \in V_{H}^{c} \subset V_{h}^{c}$ is an admissible test function in (4.8), it holds

$$
a_{h}^{I P}\left(u_{h}^{c}-u_{H}^{c}, E_{H}\left(u_{H}\right)\right)=0 .
$$

On the other hand, $u_{h}^{c}-u_{H}^{c} \in H_{0}^{2}(\Omega)$ is an admissible test function in (1.2) and hence, it holds

$$
a_{h}^{I P}\left(u, u_{h}^{c}-u_{H}^{c}\right)=\left(f, u_{h}^{c}-u_{H}^{c}\right) .
$$

We set $e_{h}^{c}:=u-u_{h}^{c}, e_{H}^{c}:=u-u_{H}^{c}$. Using (4.20), (4.21), (4.9), $h \leqslant H$, as well as Young's inequality, for the first term on the right-hand side in (4.19) we obtain

$$
\begin{aligned}
a_{h}^{I P}\left(u-E_{H}\left(u_{H}\right), u_{h}^{c}-u_{H}^{c}\right) & =\left(f, u_{h}^{c}-u_{H}^{c}\right)_{0, \Omega}=\left(f, e_{H}^{c}-e_{h}^{c}\right) \leqslant\|f\|_{0, \Omega}\left(\left\|e_{h}^{c}\right\|_{0, \Omega}+\left\|e_{H}^{c}\right\|_{0, \Omega}\right) \\
& \leqslant 2 C_{a p} H^{1 / 2+\varkappa}\|f\|_{0, \Omega}\|u\|_{5 / 2+\varkappa, \Omega} \leqslant C_{a p} H^{1 / 2+\varkappa}\left(\|f\|_{0, \Omega}^{2}+\|u\|_{5 / 2+\varkappa, \Omega}^{2}\right) .
\end{aligned}
$$

Further, in view of (1.14), (4.9), (2.4), and Young's inequality, for the second term on the right-hand side in (4.19) it follows that

$$
\begin{aligned}
a_{h}^{I P}\left(u_{H}-E_{H}\left(u_{H}\right), u_{h}^{c}-u_{H}^{c}\right) & =a_{h}^{I P}\left(u_{H}-E_{H}\left(u_{H}\right), e_{H}^{c}-e_{h}^{c}\right) \\
& \leqslant a_{h}^{I P}\left(u_{H}-E_{H}\left(u_{H}\right), u_{H}-E_{H}\left(u_{H}\right)\right)^{1 / 2} a_{h}^{I P}\left(e_{h}^{c}-e_{H}^{c}, e_{h}^{c}-e_{H}^{c}\right) \\
& \leqslant C_{1}^{1 / 2} C_{n c}^{1 / 2} \eta_{H, c}\left(\left\|e_{h}^{c}\right\|_{2, h, \Omega}+\left\|e_{H}^{c}\right\|_{2, h, \Omega}\right) \leqslant 2 C_{1}^{1 / 2} C_{n c}^{1 / 2} C_{a p} H^{1 / 2+\varkappa} \eta_{H, c}\|u\|_{5 / 2+\varkappa, \Omega} \\
& \leqslant C_{a p} H^{1 / 2+\varkappa}\left(C_{1} C_{n c} \eta_{H, c}^{2}+\|u\|_{5 / 2+\varkappa, \Omega}^{2}\right) .
\end{aligned}
$$

Finally, applying (1.14), (4.9), and Young's inequality again, the third term on the right-hand side in (4.19) can be estimated from above according to

$$
\begin{aligned}
a_{h}^{I P}\left(u_{h}-u_{H}, u_{h}^{c}-u_{H}^{c}\right) & =a_{h}^{I P}\left(u_{h}-u_{H}, e_{H}^{c}-e_{h}^{c}\right) \leqslant C_{1}\left\|u_{h}-u_{H}\right\|_{2, h, \Omega}\left(\left\|e_{h}^{c}\right\|_{2, h, \Omega}+\left\|e_{H}^{c}\right\|_{2, h, \Omega}\right) \\
& \leqslant 2 C_{1} C_{a p} H^{1 / 2+\varkappa}\left\|u_{h}-u_{H}\right\|_{2, h, \Omega}\|u\|_{5 / 2+\varkappa, \Omega} \\
& \leqslant C_{1} C_{a p} H^{1 / 2+\varkappa}\left(\left\|u_{h}-u_{H}\right\|_{2, h, \Omega}^{2}+\|u\|_{5 / 2+\varkappa, \Omega}^{2}\right) .
\end{aligned}
$$

The assertion follows from (4.22), (4.23), and (4.24).

Lemma 4.5. Under the assumptions of Theorem 4.1 it holds

$$
a_{h}^{I P}\left(u_{h}^{c}-u_{H}^{c}, u_{h}^{c}-u_{H}^{c}\right) \geqslant \frac{\gamma}{2}\left\|u_{h}^{c}-u_{H}^{c}\right\|_{2, h, \Omega}^{2}-2 C_{n c}\left(\eta_{h, c}^{2}+\eta_{H, c}^{2}\right) .
$$

Proof. Using (1.13), $u_{h}^{c}-u_{H}^{c}=u_{h}-u_{H}-\left(u_{h}^{n c}-u_{h}^{n c}\right)$, the left-hand side of the triangle inequality, and Young's inequality, we get

$$
\begin{aligned}
a_{h}^{I P}\left(u_{h}^{c}-u_{H}^{c}, u_{h}^{c}-u_{H}^{c}\right) & \geqslant \gamma\left\|u_{h}^{c}-u_{H}^{c}\right\|_{2, h, \Omega}^{2} \geqslant \gamma\left(\left\|u_{h}-u_{H}\right\|_{2, h, \Omega}-\left\|u_{h}^{n c}-u_{H}^{n c}\right\|_{2, h, \Omega}\right)^{2} \\
& =\gamma\left(\left\|u_{h}-u_{H}\right\|_{2, h, \Omega}^{2}-2\left\|u_{h}-u_{H}\right\|_{2, h, \Omega}\left\|u_{h}^{n c}-u_{H}^{n c}\right\|_{2, h, \Omega}+\left\|u_{h}^{n c}-u_{H}^{n c}\right\|_{2, h, \Omega}^{2}\right) \\
& \geqslant \frac{\gamma}{2}\left\|u_{h}-u_{H}\right\|_{2, h, \Omega}^{2}-\gamma\left\|u_{h}^{n c}-u_{H}^{n c}\right\|_{2, h, \Omega}^{2} .
\end{aligned}
$$

The assertion follows from (4.26) and Lemma 4.2.

Proof of Theorem 4.1. We have

$$
a_{h}^{I P}\left(e_{h}, e_{h}\right)=a_{h}^{I P}\left(e_{h}+u_{h}^{c}-u_{H}^{c}, e_{h}+u_{h}^{c}-u_{H}^{c}\right)-2 a_{h}^{I P}\left(e_{h}, u_{h}^{c}-u_{H}^{c}\right)-a_{h}^{I P}\left(u_{h}^{c}-u_{H}^{c}, u_{h}^{c}-u_{H}^{c}\right) .
$$

Using Lemmas 4.3, 4.4, 4.5 and observing $\varepsilon<1$, it follows that

$$
\begin{aligned}
a_{h}^{I P}\left(e_{h}, e_{h}\right) \leqslant & (1+\varepsilon) a_{H}^{I P}\left(e_{H}, e_{H}\right)-\left(\frac{\gamma}{2}-2 C_{1} C_{a p} H^{\varkappa}\right)\left\|u_{h}-u_{H}\right\|_{2, h, \Omega}^{2} \\
& +\frac{1}{\alpha \varepsilon} \frac{5\left(C_{p}+2 C_{n c}\left(1+C_{1}\right)\right)}{\gamma}\left(\eta_{h, c}^{2}+\eta_{H, c}^{2}\right)+C_{a p}\left(\left(2+C_{1}\right)\|u\|_{2+\varkappa, \Omega}^{2}+\|f\|_{0, \Omega}^{2}\right) H^{\varkappa} .
\end{aligned}
$$


We may choose $H_{0}>0$ such that for $H \leqslant H_{0}$

$$
2 C_{1} C_{a p} H^{\varkappa} \leqslant \frac{\gamma}{4}
$$

and with a constant $C_{a s}>0$, independent of $H$

$$
H^{\varkappa} \leqslant C_{a s} \hat{\eta}_{H, c}^{2}=\frac{C_{a s}}{\alpha} \eta_{H, c}^{2} \leqslant \frac{C_{a s}}{\alpha \varepsilon} \eta_{H, c}^{2} .
$$

The assertion now follows from (4.27) and (4.28a), (4.28b).

\section{Contraction property}

We now use the error reduction property (3.3), the quasi-orthogonality (4.14), and the reliability (2.5) to prove the following contraction property.

Theorem 5.1. Let $u \in H_{0}^{2}(\Omega)$ be the unique solution of (1.2). Further, let $\mathcal{T}_{h}(\Omega)$ be a simplicial triangulation obtained by refinement from $\mathcal{T}_{H}(\Omega)$, and let $u_{h} \in V_{h}, u_{H} \in V_{H}$ and $\eta_{h}, \eta_{H}$ be the $C^{0}$-IPDG solutions of (1.6) and error estimators, respectively. Then, there exist constants $0<\delta<1$ and $\rho>0$, depending only on the local geometry of the triangulations, the parameter $\Theta$ from the Dörfler marking, and on $k$, such that for sufficiently large penalty parameter $\alpha$ the fine mesh and coarse mesh discretization errors $e_{h}:=u-u_{h}$ and $e_{H}=u-u_{H}$ satisfy

$$
a_{h}^{I P}\left(e_{h}, e_{h}\right)+\rho \eta_{h}^{2} \leqslant \delta\left(a_{H}^{I P}\left(e_{H}, e_{H}\right)+\rho \eta_{H}^{2}\right) .
$$

Proof. Multiplying the estimator reduction property (3.3) by $\gamma /\left(4 C_{\tau}\right)$ and substituting the result into the quasiorthogonality (4.14), we obtain

$$
a_{h}^{I P}\left(e_{h}, e_{h}\right)+\rho \eta_{h}^{2} \leqslant(1+\varepsilon) a_{H}^{I P}\left(e_{H}, e_{H}\right)+\left(\frac{C_{Q}}{\alpha \varepsilon}-\frac{\gamma}{4 C_{\tau}}+\rho\right) \eta_{h}^{2}+\left(\frac{C_{Q}}{\alpha \varepsilon}+\frac{\gamma \varkappa(\Theta)}{4 C_{\tau}}\right) \eta_{H}^{2} .
$$

If we choose $\alpha>\left(4 C_{Q} C_{\tau}\right) /(\gamma \varepsilon)$, we have $\rho:=\gamma /\left(4 C_{\tau}\right)-C_{Q} /(\alpha \varepsilon)>0$, and it follows from (5.2) that

$$
a_{h}^{I P}\left(e_{h}, e_{h}\right)+\rho \eta_{h}^{2} \leqslant(1+\varepsilon) a_{H}^{I P}\left(e_{H}, e_{H}\right)+\left(\frac{C_{Q}}{\alpha \varepsilon}+\frac{\gamma \varkappa(\Theta)}{4 C_{\tau}}\right) \eta_{H}^{2} .
$$

Now, taking advantage of the reliability result

$$
a_{H}^{I P}\left(e_{H}, e_{H}\right) \leqslant C_{R} \eta_{H}^{2}
$$

(cf. (2.5)), for $0<\delta<1$ we obtain

$$
a_{h}^{I P}\left(e_{h}, e_{h}\right)+\rho \eta_{h}^{2} \leqslant \delta a_{H}^{I P}\left(e_{H}, e_{H}\right)+\left(C_{R}(1+\varepsilon-\delta)+\left(\frac{C_{Q}}{\alpha \varepsilon}+\frac{\gamma \varkappa(\Theta)}{4 C_{\tau}}\right)\right) \eta_{H}^{2} .
$$

We choose $\delta$ such that

$$
\rho=\frac{\gamma}{4 C_{\tau}}-\frac{C_{Q}}{\alpha \varepsilon}=\delta^{-1}\left(C_{R}(1+\varepsilon-\delta)+\left(\frac{C_{Q}}{\alpha \varepsilon}+\frac{\gamma \varkappa(\Theta)}{4 C_{\tau}}\right)\right) .
$$

Solving for $\delta$, we obtain

$$
\delta=\frac{C_{R}(1+\varepsilon)+\frac{C_{Q}}{\alpha \varepsilon}+\frac{\gamma \varkappa(\Theta)}{4 C_{\tau}}}{C_{R}+\frac{\gamma}{4 C_{\tau}}-\frac{C_{Q}}{\alpha \varepsilon}} .
$$


For instance, if we choose $\tau=\tau^{*}:=2^{-1 / 2}$ and $\varepsilon:=\gamma /\left(16 C_{R} C_{\tau^{*}}\right.$ ), we have $\varepsilon<1$ (due to $\gamma<1, C_{R}>1$, $C_{\tau^{*}}>1$ ), and, observing (3.6), it follows that

$$
\delta=\frac{C_{R}+\frac{\gamma}{16 C_{\tau^{*}}}+\frac{16 C_{Q} C_{R} C_{\tau^{*}}}{\alpha \gamma}+\frac{\gamma \Theta}{8 C_{\tau^{*}}} .}{C_{R}+\frac{\gamma}{4 C_{\tau *}}-\frac{16 C_{Q} C_{R} C_{\tau^{*}}}{\alpha \gamma}} .
$$

Looking for $\alpha$ such that

$$
\frac{\gamma}{16 C_{\tau^{*}}}+\frac{16 C_{Q} C_{R} C_{\tau^{*}}}{\alpha \gamma}+\frac{\gamma \Theta}{8 C_{\tau^{*}}}<\frac{\gamma}{4 C_{\tau *}}-\frac{16 C_{Q} C_{R} C_{\tau^{*}}}{\alpha \gamma}
$$

we find that $0<\delta<1$ for

$$
\alpha>\frac{512 C_{Q} C_{R} C_{\tau^{*}}^{2}}{(3-2 \Theta) \gamma^{2}}
$$

This concludes the proof of the contraction property.

The contraction property (5.1) is an essential ingredient to prove quasi-optimality of the adaptive approach with respect to a certain approximation class of functions depending on the regularity of the solution (cf. [6] for IPDG approximations of second order elliptic boundary value problems).

\section{Numerical results}

We provide a detailed documentation of the performance of the adaptive $C^{0}$-IPDG method for an illustrative example taken from [8].

Example 6.1. We choose $\Omega$ as the L-shaped domain $\Omega:=(-1,+1)^{2} \backslash([0,1) \times(-1,0])$ and choose $f$ in (1.1a) such that

$$
u(r, \varphi)=\left(r^{2} \cos ^{2} \varphi-1\right)^{2}\left(r^{2} \sin ^{2} \varphi-1\right)^{2} r^{1+z} g(\varphi)
$$

is the exact solution of (1.1a), (1.1b), where

$$
\begin{aligned}
g(\varphi):= & \left(\frac{1}{z-1} \sin \left(\frac{3(z-1) \pi}{2}\right)-\frac{1}{z+1} \sin \left(\frac{3(z+1) \pi}{2}\right)\right)(\cos ((z-1) \varphi)-\cos ((z+1) \varphi)) \\
& -\left(\frac{1}{z-1} \sin ((z-1) \varphi)-\frac{1}{z+1} \sin ((z+1) \varphi)\right)\left(\cos \left(\frac{3(z-1) \pi}{2}\right)-\cos \left(\frac{3(z-1) \pi}{2}\right)\right)
\end{aligned}
$$

and $z \approx 0.54448$ is a non-characteristic root of $\sin ^{2}(3 z \pi / 2)=z^{2} \sin ^{2}(3 \pi / 2)$.

For the documentation of the performance of the adaptive $C^{0}$-IPDG scheme, we have run simulations for polynomial degrees $2 \leqslant k \leqslant 6$. Since each of the constants $C_{Q}, C_{R}$, and $C_{\tau *}$ in (5.8) depends on $k^{4}$, (5.8) leads to the requirement $k^{16} \leqslant \alpha$. The numerical simulations revealed that this requirement is far too restrictive. In fact, the choice $\alpha=2.5(k+1)^{2}$ turned out to be sufficient to achieve stability and to yield optimal convergence rates. The numerical evaluation of the element residuals has been taken care of by the collapsed GaussJacobian-type quadrature formulas from [23] which worked fine even for triangles containing the origin as a vertex.

For $k=2,4,6$, Figures $1-6$ show the adaptively refined meshes after 10 adaptive cycles (top left), the convergence histories in terms of the broken $C^{0}$-IPDG energy norm of the error $a_{h}^{I P}\left(u-u_{h}, u-u_{h}\right)^{1 / 2}$ as a function of the total number of degrees of freedom (DOF) on a logarithmic scale (top right), the decrease of the estimator as a function of the DOF (bottom left), as well as the computed effectivity indices $\eta_{h} / a_{h}^{I P}\left(u-u_{h}, u-u_{h}\right)^{1 / 2}$ (bottom right) for uniform refinement and adaptive refinement with $\Theta=0.7$ and $\Theta=0.3$ in the Dörfler marking. 

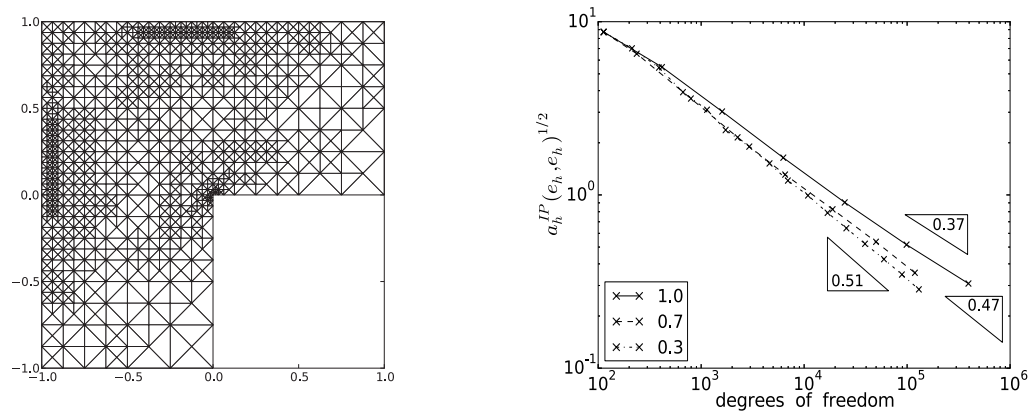

Figure 1. $k=2$ : Refined mesh after 10 adaptive cycles (left) and convergence history (right).
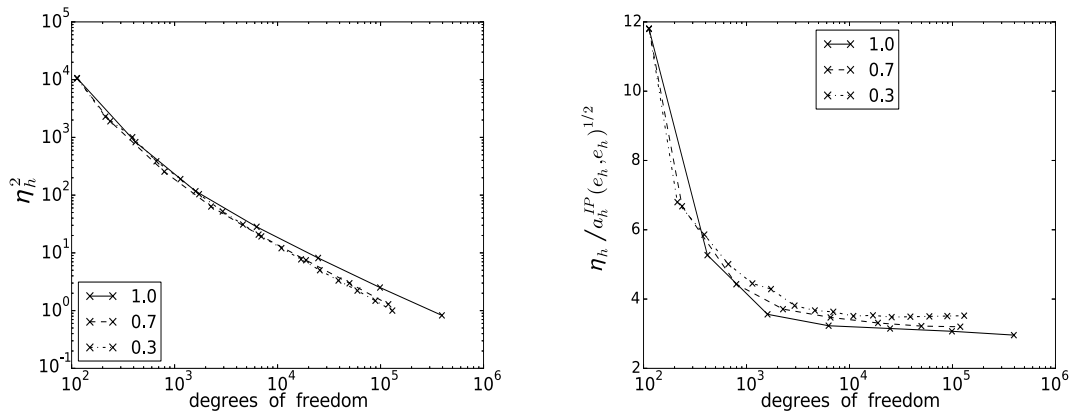

Figure 2. $k=2$ : Estimator reduction (left) and effectivity indices (right).
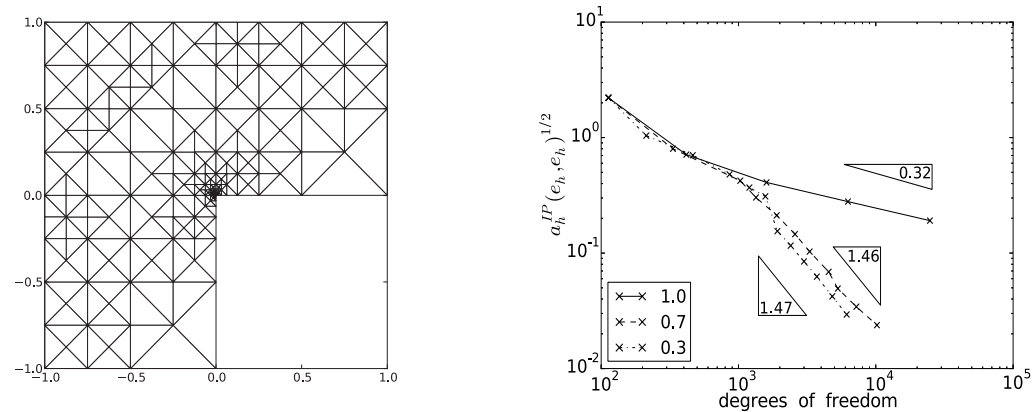

Figure 3. $k=4$ : Refined mesh after 10 adaptive cycles (left) and convergence history (right).
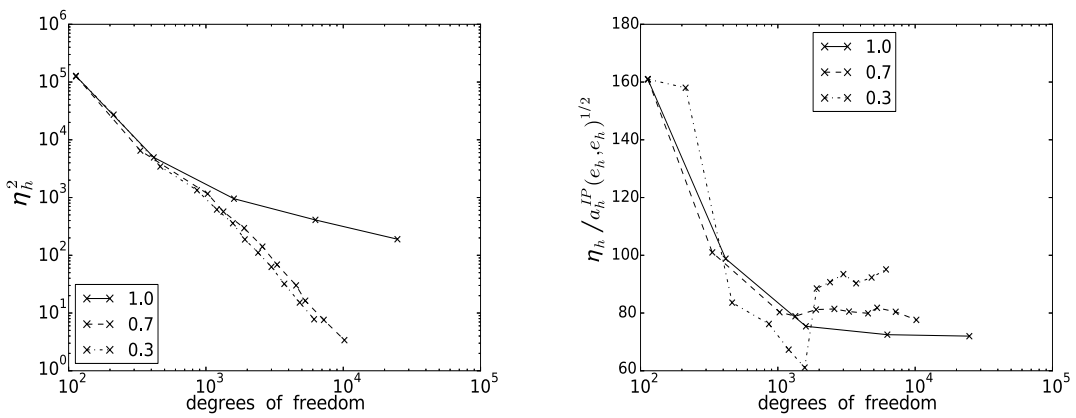

Figure 4. $k=4$ : Estimator reduction (left) and effectivity indices (right). 

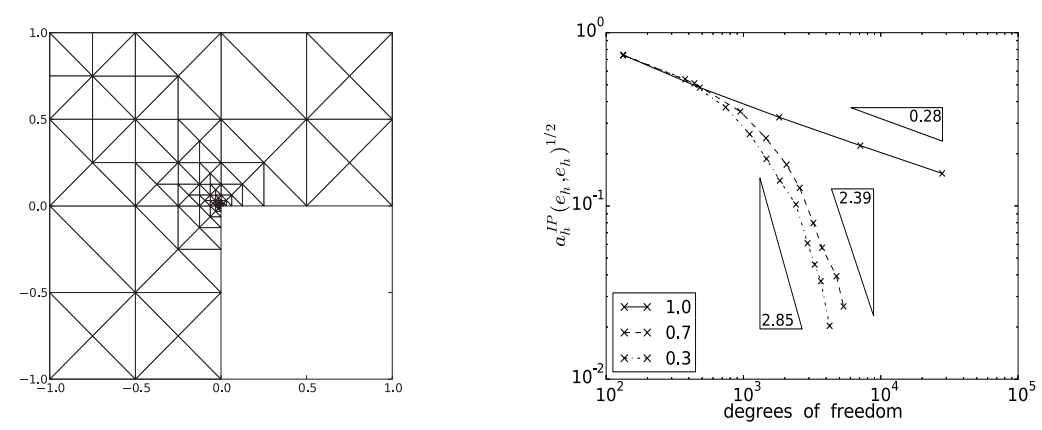

Figure 5. $k=6$ : Refined mesh after 10 adaptive cycles (left) and convergence history (right).
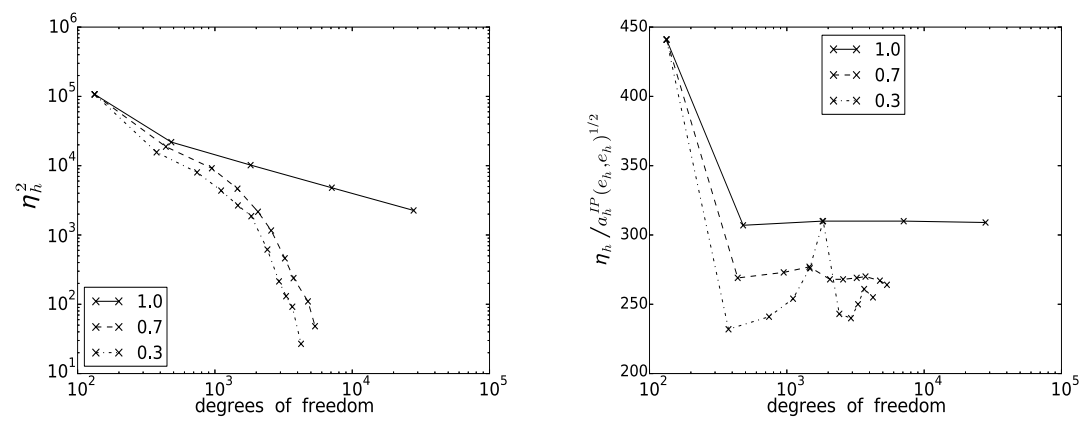

Figure 6. $k=6$ : Estimator reduction (left) and effectivity indices (right).

As has been shown in [9], we have

$$
a_{h}^{I P}\left(u-u_{h}, u-u_{h}\right)^{1 / 2} \leqslant\left(\sum_{T \in \mathcal{T}_{h}(\Omega)}(\operatorname{diam}(T))^{2 \min (\alpha(T), k-1)}|u|_{2+\alpha(T), T}^{2}\right)^{1 / 2}
$$

where $\alpha(T), T \in \mathcal{T}_{h}(\Omega)$, is the local index of elliptic regularity. We note that $\min (\alpha(T), k-1)=z \approx 0.544$ for elements $T$ having a vertex at the origin and $\min (\alpha(T), k-1)=k-1$ elsewhere. Consequently, the expected optimal convergence rates are slightly less than 0.5 for $k=2,1.5$ for $k=4$, and 2.5 for $k=6$. Figures 1 (right), 3 (right), and 5 (right) show that these optimal convergence rates are asymptotically achieved by the adaptive algorithm. Moreover, as in case of IPDG methods for second order elliptic boundary value problems [19] and H-IPDG methods for Maxwell's equations [12] we observe a different convergence behavior depending on the choice of $\Theta$ in the Dörfler marking. The effectivity indices show a clear dependence on the polynomial degree $k$.

Funding: The second author has been supported by NSF grants DMS-0707602, DMS-0810176, DMS-0811153, DMS-0914788, by the DFG within the Priority Program SPP 1253, by the BMBF within the Collaborative Research Project 'MeFreSim', and by the ESF within the Research Networking Programme 'OPTPDE'. The second and the third author have been further supported by the BMBF within the Collaborative Research Project 'FROPT'. All authors acknowledge support by the DFG within the Priority Program SPP 1506.

\section{References}

[1] M. Ainsworth and J. T. Oden, A Posteriori Error Estimation in Finite Element Analysis. Wiley, Chichester, 2000.

[2] J. H. Argyris, I. Fried, and D. W. Scharpf, The TUBA family of plate elements for the matrix displacement method. Aero. J. Roy. Aero. Soc. 72 (1968), 701-709.

[3] I. Babuska and T. Strouboulis, The Finite Element Method and its Reliability. Clarendon Press, Oxford, 2001. 
[4] W. Bangerth and R. Rannacher, Adaptive Finite Element Methods for Differential Equations. Lectures in Mathematics. ETHZürich. Birkhäuser, Basel, 2003.

[5] P. Binev, W. Dahmen, and R. DeVore, Adaptive finite element methods with convergence rates. Numer. Math. 97 (2004), 219-268.

[6] A. Bonito and R. Nochetto, Quasi-optimal convergence rate of an adaptive Discontinuous Galerkin method. SIAM J. Numer. Anal. 48 (2010), 734-771.

[7] S. C. Brenner and L. R. Scott, The Mathematical Theory of Finite Element Methods. Springer, New York, 2008.

[8] S. C. Brenner, T. Gudi, and L.-Y. Sung, An a posteriori error estimator for a quadratic $C^{0}$-interior penalty method for the biharmonic problem. IMA J. Numer. Anal. 30 (2010), 777-798.

[9] S. C. Brenner and L.-Y. Sung, $C^{0}$ interior penalty methods for fourth order elliptic boundary value problems on polygonal domains. J. Sci. Comput. 22/23 (2005), 83-118.

[10] S. C. Brenner, K. Wang, and J. Zhao, Poincaré-Friedrichs inequalities for piecewise $H^{2}$ functions. Numer. Funct. Anal. Optim. 25 (2004), 463-478.

[11] C. Carstensen and R. H. W. Hoppe, Convergence analysis of an adaptive nonconforming finite element method. Numer. Math. 103 (2006), 251-266.

[12] C. Carstensen, R. H. W. Hoppe, N. Sharma, and T. Warburton, Adaptive hybridized Interior Penalty Discontinuous Galerkin methods for H(curl)-elliptic problems. Numer. Math. Theor. Meth. Appl. 4 (2011), 13-37.

[13] J. M. Cascon, Ch. Kreuzer, R. H. Nochetto, and K. G. Siebert, Quasi-optimal rate of convergence of adaptive finite element methods. SIAM J. Numer. Anal. 46 (2008), 2524-2550.

[14] W. Dörfler, A convergent adaptive algorithm for Poisson's equation. SIAM J. Numer. Anal. 33 (1996), 1106-1124.

[15] G. Engel, K. Garikipati, T. J. R. Hughes, M. G. Larson, L. Mazzei, and R. L. Taylor, Continuous/discontinuous finite element approximations of fourth order elliptic problems in structural and continuum mechanics with applications to thin beams and plates, and strain gradient elasticity. Comput. Methods Appl. Mech. Engrg. 191 (2002), 3669-3750.

[16] K. Eriksson, D. Estep, P. Hansbo, and C. Johnson, Computational Differential Equations. Cambridge University Press, Cambridge, 1995.

[17] E. H. Georgoulis and P. Houston, Discontinuous Galerkin methods for the biharmonic problem. IMA J. Numer. Anal. (2009) 29, 573-594.

[18] E. H. Georgoulis, P. Houston, and J. Virtanen, An a posteriori error indicator for discontinuous Galerkin approximations of fourth order elliptic problems. IMA J. Numer. Anal. 31 (2011), 281-298.

[19] R. H. W. Hoppe, G. Kanschat, and T. Warburton, Convergence analysis of an adaptive interior penalty discontinuous Galerkin method. SIAM J. Numer. Anal. 47 (2009), 534-550.

[20] P. Houston, D. Schötzau, and T. Wihler, Mixed $h p$-discontinuous Galerkin methods for the Stokes problem in polygons. In: Numerical Mathematics and Advanced Applications, ENUMATH 2003 (Eds. M. Feistauer et al.) Springer, BerlinHeidelberg-New York, 2004, pp. 483-492.

[21] P. Houston, D. Schötzau, and T. Wihler, Energy norm a posteriori error estimation of hp-adaptive discontinuous Galerkin methods for elliptic problems. Math. Models Methods Appl. Sci. 17 (2007), 33-62.

[22] O. Karakashian and F. Pascal, Convergence of adaptive discontinuous Galerkin approximations of second-order elliptic problems. SIAM J. Numer. Anal. 45 (2007), 641-665.

[23] G. E. Karniadakis and S. J. Sherwin, Spectral/hp Methods for CFD. Oxford University Press, Oxford-New York, 1999.

[24] P. Morin, R. H. Nochetto, and K. G. Siebert, Data oscillation and convergence of adaptive FEM. SIAM J. Numer. Anal. 38 (2000), No. 2, 466-488.

[25] P. Neittaanmäki and S. Repin, Reliable Methods for Mathematical Modelling. Error Control and a posteriori Estimates. Elsevier, New York, 2004.

[26] D. Schötzau, C. Schwab, and A. Toselli, Mixed hp-dgfem for incompressible flows. SIAM J. Numer. Anal. 40 (2003), 21712194.

[27] L. Tartar, Introduction to Sobolev Spaces and Interpolation Theory. Springer, Berlin-Heidelberg-New York, 2007.

[28] R. Verfürth, A Posteriori Estimation Techniques for Finite Element Methods. Oxford University Press, Oxford, 2013.

[29] T. Warburton and J. S. Hesthaven, On the constants in $h p$-finite element trace inverse inequalities. Comput. Methods Appl. Mech. Engrg. 192 (2003), 2765-2773.

[30] G. N. Wells, K. Garikipati, and L. Molari, A discontinuous Galerkin formulation for a strain gradient dependent damage model. Comput. Methods Appl. Mech. Engrg. 193 (2004), 3633-3645. 
Copyright of Journal of Numerical Mathematics is the property of De Gruyter and its content may not be copied or emailed to multiple sites or posted to a listserv without the copyright

holder's express written permission. However, users may print, download, or email articles for individual use. 